\title{
Addition, Subtraction, and Analysis of Orbital Angular Momentum in Electron Vortex Beams
}

Tyler S. Yahn, Jordan S. Pierce, Tyler R. Harvey, Benjamin J. McMorran

Department of Physics, University of Oregon, Eugene, OR 97405, USA

In this work we present a method for analyzing the quantized orbital angular momentum (OAM) of a free electron vortex beam [1,2,3] (Figure 2) using a holographic diffraction grating similar to that previously described by our group [4]. We use this nanoscale grating to imprint additional OAM onto an incident beam, as shown in Figure 3. When using a vortex beam with OAM incident upon this grating, the OAM of a diffracted electron beam is the sum of the input beam's OAM and the OAM imparted by the grating. However, we find that the total intensity in each diffraction order is unaffected by the OAM in the incident beam, which has consequences for previous interpretations of results from gratings used as mode analyzers [3].

This work was conducted using an FEI Titan TEM operating with at $300 \mathrm{kV}$. The electron beam was passed through a $\varnothing 50 \mu \mathrm{m}$ diffraction grating with a single fork dislocation placed in the TEM's second condenser aperture. The resulting first order diffracted from this grating with an imprinted OAM with a magnitude of $1 \hbar$ was then used to solely illuminate a similarly constructed $\varnothing 5 \mu \mathrm{m}$ diffraction grating located in the TEM's sample holder. The diffraction from the sample plane grating was then recorded on the TEM's CCD camera.

Figure 1 illustrates addition of OAM to the incident vortex beam. In both images we have used a nanofabricated holographic grating with a single fork dislocation. The quantized unit of OAM added or subtracted in each diffraction order of the holographic grating was made to be the same as the incident electron vortex beam's OAM, namely $1 \hbar$. We have varied the relative chirality of the input vortex beam with respect to the fork dislocation such that the diffraction beams have opposite phase singularities in nonzero orders. As shown in Figure 1A, the OAM added to the +1 diffraction order on the right has canceled the incident vortex beam's OAM and the resulting beam has no OAM. In contrast the OAM added to the -1 diffraction order on the left has increased the magnitude of the beams OAM to $2 \hbar$. This pattern of adding (subtracting) OAM on the left (right) continues for the higher order diffracted beams. Figure 1B shows the opposite behavior of Figure 1A in that the higher order diffraction beams to the right (left) add (subtract) OAM from the incident beam which now has an opposite chirality.

Analysis of our findings [Table 1] show the intensities of the first order diffracted beams are unaffected by the incident beams OAM. Table 1 shows the ratio of the integrated density of the +1 order diffraction to the -1 order for the opposite OAM input vortex beams remain within measured error of each other. Furthermore, we measured no differences in these ratios greater than $0.4 \%$. As such these findings are not congruent with the analysis presented by Verbeek et al [3].

\section{References}

[1] M. Uchida, A. Tonomura, Nature 464 (2010) 737.

[2] B. J. McMorran et al, Science 331 (2011) p. 192. 
[3] J. Verbeeck, H. Tian and P. Schattschneider, Nature 467 (2010) p. 301.

[4] B. J. McMorran et al, Microsc. Microanal. 17 (Suppl 2) (2011) p. 1236
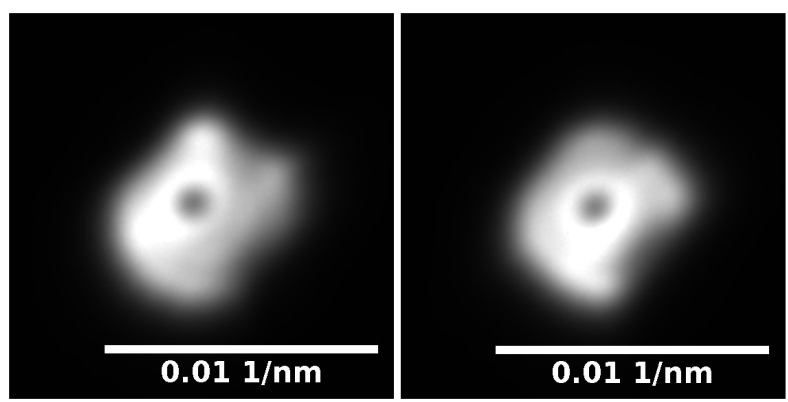

Figure 2: Images of the -1 (left) and +1 (right) incident electron vortex beams each having defined OAM magnitude of $1 \hbar$.
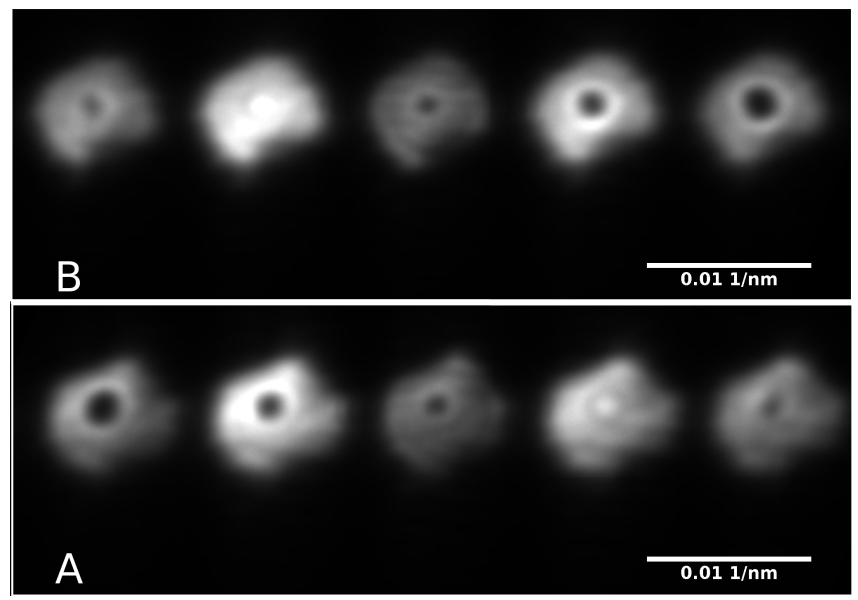

Figure 1: Images of an incident vortex beam having 1 unit OAM diffracted through a single forked grating in the objective plane. The chirality of the incident beam used in (A) is opposite as that in (B). Both images are taken consecutively with the same beam settings.

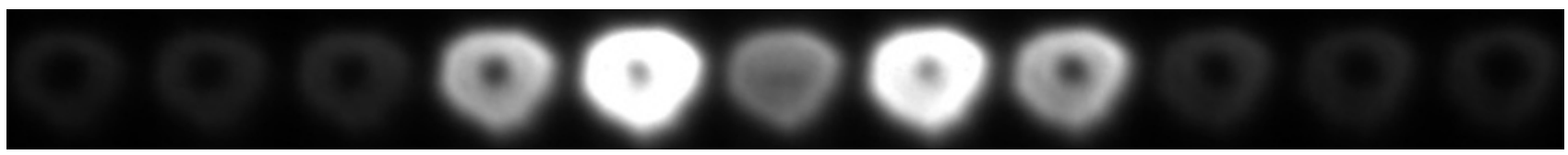

Figure 3: Diffraction image of the objective plane grating illuminated by a plane wave. The spot in the center is the $0^{\text {th }}$ order diffraction beam with $0 \hbar$ OAM. The higher order beams on either side of the $0^{\text {th }}$ order beam clearly show increasing or decreasing amounts of OAM as expected.

\begin{tabular}{|c|c|}
\hline Chirality Relation & Measured Ratio \\
\hline Positive & $1.201+/-0.001$ \\
\hline Negative & $1.2027+/-0.0009$ \\
\hline
\end{tabular}

Table 1: Measured ratio of the +1 to the -1 diffraction orders for data similar to that shown in Figure 1. The values are found by taking multiple measurements of the integrated density of the +1 and -1 diffraction orders over a similar area. The chirality relation is defined such that positive is the relation shown in Figure 1A and negative in Figure 1B. 